\title{
La imagen como recurso estratégico en la gestión de instituciones hoteleras: el caso del Hotel Kohly de la Ciudad de La Habana
}

DOI: $10.22403 /$ UQROMX/TYP10/02

\author{
Dayana Duffus Miranda \\ Roberto Carmelo Pons \\ Facultad de Ciencias Económicas \\ Universidad Central "Marta Abreu" de Las Villas
}

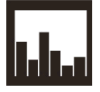

\section{RESUMEN}

La industria turística enfrenta hoy nuevos retos, esto conlleva la necesidad de reinventar la gestión empresarial, utilizando para ello variables determinantes en el sector turístico, como la imagen. El presente trabajo aborda la imagen como una fuente generadora de ventajas competitivas para la planta hotelera en Cuba, así como su papel dentro de la dirección estratégica de los hoteles, y tiene como objetivo proponer un procedimiento que permita medir la imagen de hoteles en Cuba para una gestión eficaz de los sistemas de servicios que los integran. Para ello se desarrolla una investigación de mercado que permite definir la imagen general del Hotel Kohly y de sus componentes; determinando la satisfacción de los clientes y su disposición a repetir la visita. Se utilizan técnicas cuantitativas y cualitativas, que incluyen la observación directa, la entrevista a profundidad y la encuesta personal. De esta forma se da respuesta a los objetivos planteados y se contrastan las hipótesis de partida. En el marco del perfeccionamiento empresarial cubano, el procedimiento propuesto para medir la imagen contribuye a lograr en el sector turístico empresas aptas para usar satisfactoriamente sus recursos y capacidades desde la perspectiva de la demanda.

Palabras Componente afectivo, componente cognitivo, componente único,

CLAVE dirección estratégica, imagen de hoteles, satisfacción

Recibido el 2 de septiembre de 2010

Aprobado el 29 de octubre de 2010

Correos electrónicos: dduffusm@uclv.edu.cu•rpons@uclv.edu.cu 


\section{Introducción}

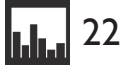

\section{2}

La industria turística ha presentado un alto nivel de crecimiento en la última década. De acuerdo con los informes del Departamento de Estadística de la Organización Mundial del Turismo (OMT), la industria está en pleno crecimiento: en 1960 se registraron 70 millones de llegadas en el nivel mundial; en 1980, 286 millones; en 2000, 659 millones, y se preveía que para 2010 serían mil millones.

El sector turístico no sólo se enfrenta a un crecimiento muy dinámico, sino que también existen nuevos desafíos: cambios acelerados en el entorno económico, político y tecnológico; disminución drástica de las distancias, de los tiempos de reacción; riesgos de deterioro en áreas urbanas; creciente número de competidores; mayor disponibilidad de información por parte de los compradores, y dependencia cada vez mayor de recursos propios; entonces, surge la necesidad de pensar globalmente, pero actuar localmente. Ante estas exigencias del entorno empresarial turístico, el administrador debe reinventar la gestión corporativa mediante la utilización de las más variadas herramientas, una de las cuales es la imagen institucional. La imagen como elemento de administración desempeña un papel determinante en el turismo. Esto se debe a que la actitud de un consumidor hacia un producto turístico es resultado de la percepción, reflejo de la imagen, que se ha formado del mismo.

El objetivo general de este trabajo es proponer un procedimiento que permita medir la imagen de hoteles en Cuba para una gestión eficaz de los sistemas de servicios que los integran. Se parte de una secuencia metodológica deductiva donde se realiza una exhaustiva revisión bibliográfica relativa a la definición de la imagen de hoteles y al papel de ésta en el marco de la dirección estratégica. Se valora también este cuerpo de conocimientos con el propósito de identificar secuencias de análisis comunes, asociación de estructuras complementarias que, haciendo uso de la síntesis, nos permitan establecer el estado de la cuestión respecto a la conceptualización, papel y medición de la imagen en la gestión y comercialización de hoteles, así como de sus variables asociadas.

Este análisis confirma la necesidad de proponer un procedimiento de medición de imagen, para lo cual utilizaremos una secuencia metodológica de orientación inductiva, que requiere, en primer lugar, partir de postulados teórico-prácticos establecidos de acuerdo con la imagen, su relación con otras variables y su papel dentro de la gestión y comercialización de servicios de hoteles. En segundo lugar, el empleo de técnicas de investigación cuantitativas y cualitativas que permitan desarrollar el procedimiento propuesto. 


\section{Definición del constructo imagen para su correcta medición}

En la actualidad, la palabra imagen es usada para definir gran cantidad de

fenómenos. Esto se debe, principalmente, a la polisemia del término. Tal profusión de significados ha hecho que su empleo en el ámbito empresarial sea confuso, y que los aspectos a medir al referirse a la imagen sean difusos y muy abstractos. Por lo tanto, si vamos a medir imagen de empresas, debemos partir de su correcta definición: la imagen de hoteles es un concepto que se forma a través de la interpretación razonada y emocional del consumidor, y es consecuencia de la combinación de dos componentes estrechamente interrelacionados: el perceptual-cognitivo y el holístico (San Martín Gutiérrez, 2005: 68; Beerli, Martín y Moreno, 2008: 7). El primero está integrado por características funcionales y psicológicas que identifican al hotel; éstas dividen los atributos de la imagen en directamente observables y medibles (funcionales), e intangibles y más difíciles de observar y medir (psicológicos).

Por su parte, el componente holístico está constituido por el conjunto de emociones que suscita la empresa de alojamiento, concibiendo la realidad como un todo distinto de la suma de las partes que lo componen. Se basa en las investigaciones concernientes a la naturaleza del procesamiento de la información por el ser humano, desde los campos de la psicología y el comportamiento del consumidor. Es decir, cada producto es percibido tanto en términos de "características funcionales propias" como de "impresiones o conjunto de imágenes". Para una medición completa debemos añadir el componente único distintivo, constituido por las características que lo hacen diferente de un hotel concreto. Sin duda, la imagen es un constructo complejo que sólo se puede medir sobre la base de los componentes que lo integran.

\section{La imagen como ventaja competitiva en la dirección estratégica de los hoteles cubanos}

La dirección estratégica es un proceso que se encamina hacia la consecución y mantenimiento de ventajas competitivas que permitan a la empresa continuar en el mercado. En el caso de los hoteles, debemos partir de que no se puede comprar el servicio a priori sino la imagen que lo precede. Esto se debe a las peculiaridades de este tipo de servicio, a su intangibilidad, pues los servicios 
no se pueden experimentar en los sentidos antes de su compra; quien los suministra debe gestionar la evidencia: hacer tangible lo intangible. Por otro lado, su heterogeneidad y agregabilidad, así como la distancia física y temporal entre la decisión y el consumo, conllevan que, para los productos turísticos, lo más importante por comercializar sean imágenes. Numerosos estudios empíricos han confirmado el papel de la percepción como elemento causal en el proceso de creación de las imágenes que los individuos atribuyen a los lugares turísticos, y como uno de los factores condicionantes de la decisión de compra (Gándara,Torres Bernier y Costa Mielke, 2007). Esta imagen se extiende desde la fase previa a la visita al lugar, pasando por la evaluación posterior tras su estancia, hasta afectar las intenciones futuras de comportamiento (Chen y Pan, 2006: 60; Pons y Morales, 2008: 3). Las firmas precisan comprender el comportamiento de sus consumidores, sus necesidades y actitudes, y diseñar su oferta en correspondencia con la imagen a proyectar para que sea atractiva y satisfaga los requerimientos del mercado. En tal sentido, la imagen (resultado de una multiplicidad de causas) constituye una de las ventajas competitivas en el sector turístico que genera mayor valor.

El administrador debe tener conocimiento de su organización desde la perspectiva del consumidor; la medición de la imagen se convierte en un arma potente para realizar los ajustes precisos del lado de la oferta -pero desde una visión de la demanda-, lo que garantiza el triunfo de la industria turística en el contexto de un mercado de fuerte competencia.

Una característica de la cultura empresarial de Cuba es la falta de regularidad en las evaluaciones de la dinámica de los mercados, lo cual limita el desarrollo de nuevas capacidades empresariales relacionadas con la introducción de originales formas de organización y dirección de la economía en las empresas, lo que incluye aquellas medidas encaminadas a asegurar la calidad de la producción: actualización de normas y mejoramiento de relaciones con los proveedores y clientes, entre otras. Cabría añadir que las empresas cubanas enfrentan problemas para orientarse hacia la competitividad (Pérez Villanueva, 2004: 64). La propia evolución dinámica del contexto de referencia y de pertenencia de la organización, el entorno competidor y los gustos y preferencias de los públicos objetivo, exigen la readaptación dinámica de los atributos de la imagen. El perfeccionamiento empresarial llevado a cabo en Cuba establece en su filosofía general que la empresa cubana debe velar por el 


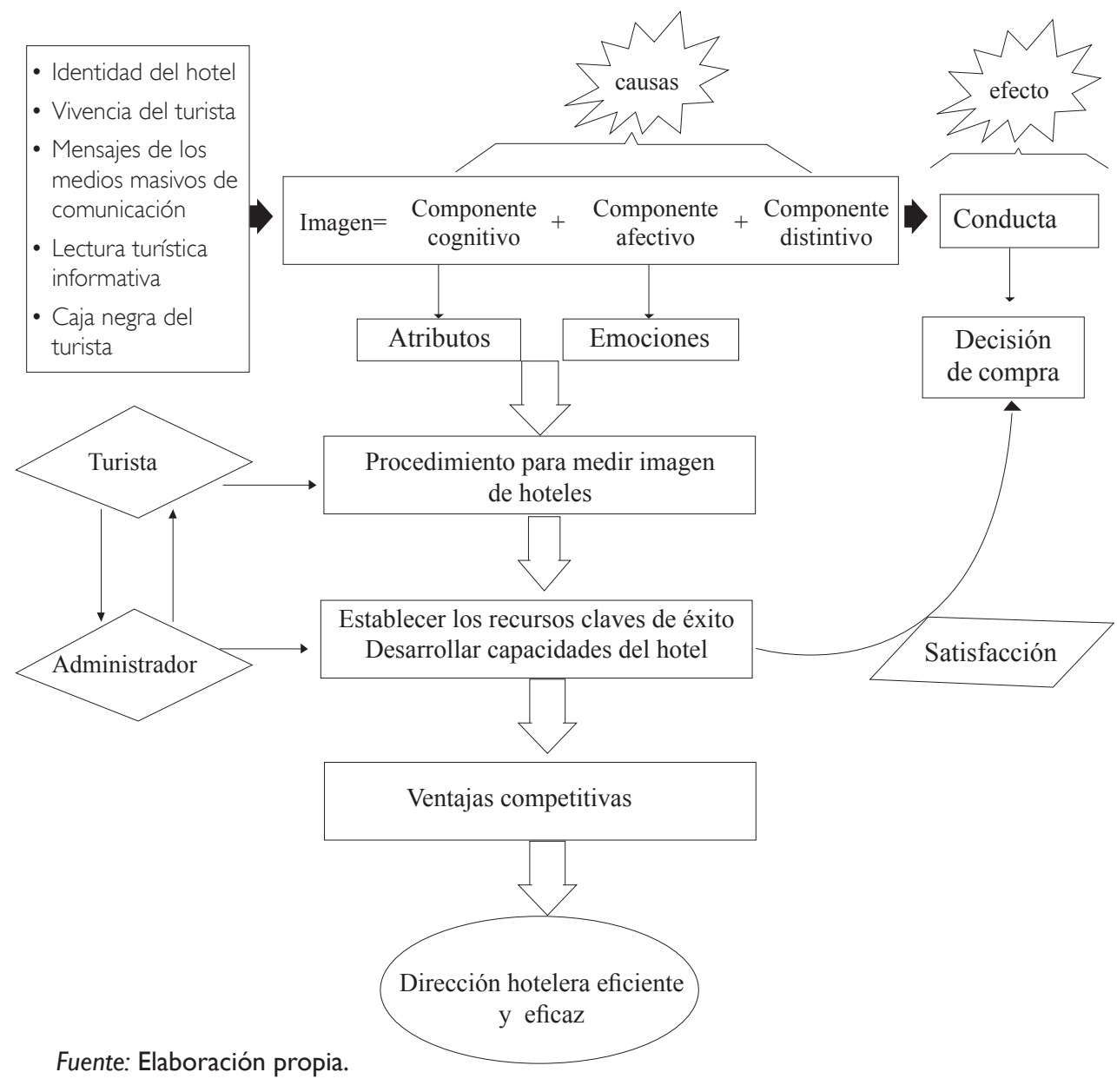

Figura I. Modelo conceptual: la imagen como recurso y Capacidad en la ADMINISTRACIÓN DE LA EMPRESA HOTELERA TURÍSTICA

empleo óptimo de cada recurso; si cada insumo no está en función de satisfacer las necesidades y deseos de cada turista, como determinante en última instancia de los beneficios a largo plazo, no será posible dar un uso más racional a estos recursos en función. Lo anterior sustenta la necesidad de desarrollar en la 
industria cubana de alojamiento turístico un procedimiento capaz de medir la imagen como elemento determinante en el diseño de los sistemas de servicio de la planta hotelera, teniendo en cuenta la visión de sus clientes.

\section{Propuesta de procedimiento para medir la imagen de hoteles en Cuba}

Con base en los aspectos conceptuales analizados y establecidos es menester, en primera instancia, precisar que la imagen de un hotel no se puede cuantificar de manera directa; los atributos con que se puede medir no son claramente observables en todos los casos, e inclusive pueden diferir entre sujetos (Baloglu y McCleary, 1999: 868; Pons, 2000: 23; Díaz Armas, 2003: 28; Beerli, Martín y Moreno, 2008: 7); al proponerse medirlos debe conseguirse una estructura de relaciones que resulte cuantificable, donde desempeña un papel cardinal el establecimiento de escalas que discriminen entre criterios, así como la utilización de la filosofía propia de la matriz mantilla y la teoría multiatributo (Varela, Rial y García-Cueto, 2003:656; Chen y Pan, 2006:60). Hasta el momento, la combinación de varias técnicas es lo más adecuado, considerando los aportes de la investigación cuantitativa y cualitativa (Sanz de la Tajada, 1994:60; Baloglu y McCleary, 1999:868; Pons, 2000:23; Chu y Choi, 2000: 363; Chen y Pan, 2006: 60; Beerli, Martín y Moreno, 2008: 7).A esto hay que añadir que la metodología debe corresponderse con la conceptualización de imagen de hotel, lo cual nos obliga a tener presente los diferentes aspectos que caracterizan el concepto de imagen; a saber:

a) La conceptualización de la imagen de un hotel como resultado de las percepciones y actitudes del potencial cliente (Baloglu y McCleary, 1999: 868; Pons, 2000: 23; Díaz Armas, 2003: 28; Chen y Pan, 2006: 60), razón que justifica la combinación de técnicas cualitativas y cuantitativas y que redundará en un procedimiento más completo.

b) La gran variedad y complejidad de los atributos que conforman el constructo imagen de un hotel (Chu y Choi, 2000: 363; Díaz Armas, 2003: 28;Wai-Ching y Gun-Fie, 2005; Beerli, Martín y Moreno, 2008: 7), lo que implica que la técnica estadística de tratamiento y análisis de la información debe ser multivariable (Wai-Ching y Gun-Fie, 2005), pues 
la diversidad de componentes subjetivos y objetivos obliga a medir atributos funcionales y psicológicos, además de considerar elementos holísticos, únicos y distintivos (Baloglu y McCleary, 1999: 868; Costa, 2003: 10; Díaz Armas, 2003: 28; Beerli, Martín y Moreno, 2008: 7).

c) El carácter dinámico y cambiante de la imagen en el tiempo según el entorno (Sanz de la Tajada, 1994: 60; Díaz Armas, 2003: 28; Chen y Pan, 2006: 60).

Esta necesidad metodológica nos ha llevado a crear un procedimiento específico basado en un paradigma sistémico y holístico. Si la imagen es la ciencia de la totalidad, el método para responder a esta cuestión no podía ser sino global, holístico (Costa, 2003: 10).

El principal aporte de este procedimiento consiste en la integración coherente y orgánica de las perspectivas de la empresa y del cliente en la evaluación de la imagen del servicio, que derive en la mejora del proceso de prestación del servicio y en el cumplimiento de las expectativas del cliente, logrando un uso eficiente y eficaz de los recursos y capacidades de la planta hotelera.

El procedimiento que se propone a continuación, capaz de medir de forma válida y fiable la imagen de hoteles en Cuba, se desarrolla en relación con los componentes antes abordados como integrantes de la imagen de hoteles. Para su análisis se parte de la metodología planteada por Sanz de la Tajada (1994: 60 ), que considera las fases definidas referidas al estudio de gabinete, una fase cualitativa y otra cuantitativa.

El procedimiento propuesto está conformado por los siguientes nueve pasos, agrupados en tres fases:

Fase I. Definición del problema a resolver

I. Caracterización del objeto de estudio

2. Establecimiento de objetivos de la medición de imagen

3. Determinación de hipótesis de investigación

Fase II. Elaboración del instrumento de investigación

4. Generación de atributos que forman parte de los componentes de la imagen de hoteles

5. Diseño del instrumento de investigación: el cuestionario

6. Pretest del cuestionario 
Fase III. Trabajo de campo y presentación de los resultados de la investigación

7. Recolección de la información

8. Procesamiento e interpretación de la información

9. Informe final del estudio de imagen

Fase I. Definición del problema a resolver

La fase I comienza con la caracterización del objeto de estudio (paso I). Éste incluye la caracterización general del hotel:su ubicación geográfica, cadena a la que pertenece, nivel de ocupación promedio por temporada, capacidad de la planta hotelera, así como un análisis de la situación económica financiera de la instalación en los últimos tres años. Todos estos datos permitirán al investigador diagnosticar la situación actual de cada hotel donde se proponga medir la imagen.A esto le sigue el paso 2: establecimiento de objetivos de investigación, asumiendo el concepto de imagen que va a regir todo el estudio, lo que hará posible definir los resultados que se pretenden alcanzar, guía indispensable para el resto de las fases. El paso 3 consiste en la determinación de las hipótesis de investigación, las cuales se tratará de demostrar mediante la conducción de la investigación empírica, como prueba de una evidencia.Todo esto lleva a una segunda fase dentro de la investigación.

Fase II. Elaboración del instrumento de investigación

La fase II tiene como objetivo principal elaborar el instrumento que servirá para medir la imagen de los hoteles en Cuba. Esta fase inicia con el paso 4, donde se generan, en un primer momento, los atributos que forman parte de los componentes de la imagen de los hoteles a partir de una amplia revisión de la literatura especializada. Para esto se parte de la correcta definición de los componentes que integran la imagen. En la literatura es ampliamente abordado el concepto de imagen como un constructo muy complejo (Sanz de la Tajada, 1994: 60; Costa, 2003: I0; Pons, 2000: 23; Díaz Armas, 2003: 28; Chen y Pan, 2006: 60; Beerli, Martín y Moreno, 2008: 7), fundado en que la parte más avanzada de la configuración mental de la imagen fija una relación con el estado objetivo y real de las cosas. Sin duda, es una relación subjetivada, o sea, progresivamente constituida, porque la imagen no es resultado de un impacto instantáneo; es un proceso psicológico casi inconsciente, que se devela 
y aparece en un momento dado (Costa, 2003: 10). Esto significa la existencia de una diversidad de componentes a considerar en un análisis de la imagen, sin que haya unanimidad por parte de los investigadores.

La definición aceptada de medición de imagen de un hotel nos sugiere que una completa instrumentación incluye la medición de los componentes perceptual-cognitivo, holístico y único distintivo (Pons, 2000: 23; Díaz Armas, 2003: 28; Beerli, Martín y Moreno, 2008: 7).

El componente holístico en numerosos estudios es relegado a un segundo plano o no es tratado (Chu y Choi, 2000; Wai-Ching y Gun-Fie, 2005; Ryan y Huimin, 2007), a pesar de que diversos autores, como Pearce (1982: 10), Echtner y Ritchie ( 1993:3) o Baloglu y Brinberg ( 1997: I I), Díaz Armas (2003: 30) y Beerli, Martín y Moreno (2008: 7), se hayan referido a su importancia. En la figura 2 se muestran graduaciones utilizadas al medir el componente holístico de la imagen.

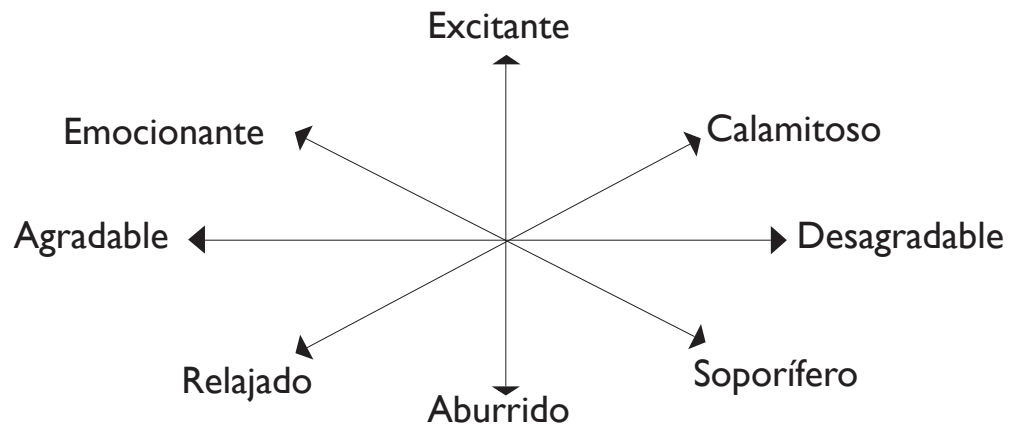

Fuente: Baloglu y Brinberg, 1997: 15.

Figura 2. Modelo de representación en dos dimensiones del componente holístico. Atributos para lugares

Estudios relacionados con el caso particular de hoteles utilizan estas emociones añadiendo seguridad, confort e inspiración (Barsky y Nash, 2003: 7). Diversas investigaciones han demostrado que las emociones juegan un papel muy importante en la satisfacción de los clientes y su lealtad hacia el hotel (Akin, 2006: I87). 
La imagen como recurso estratégico en la gestión de instituciones hoteleras: el caso del Hotel Kohly de la Ciudad de La Habana

Cuadro I. Atributos más relevantes para medir la imagen de un hotel

\begin{tabular}{|c|c|}
\hline Atributos & Referenciado por el autor \\
\hline Limpieza & $\begin{array}{l}\text { Peiró, Martínez-Tur y Ramos López, I999; Kandampully y } \\
\text { Suhartanto, 2000; Varela, Rial y García-Cueto, 2003; Chu y Choi, } \\
\text { 2000; Wai-Ching y Gun-Fie, } 2005 .\end{array}$ \\
\hline Seguridad & $\begin{array}{l}\text { Peiró, Martínez-Tur y Ramos López, 1999; Chu y Choi, 2000; } \\
\text { Wai-Ching y Gun-Fie, 2005;Aksu, 2006; Ryan y Huimin, } 2007 .\end{array}$ \\
\hline $\begin{array}{l}\text { Valor } \\
\text { alojamiento/ } \\
\text { precio }\end{array}$ & $\begin{array}{l}\text { Kandampully y Suhartanto, 2000; Wai-Ching y Gun-Fie, 2005; } \\
\text { Jonasson y Mård, } 2007 .\end{array}$ \\
\hline Empleados & $\begin{array}{l}\text { LeBlanc y Nguyen, 1996; Peiró, Martínez-Tur y Ramos López, I999; } \\
\text { Kandampully y Suhartanto, 2000; Chu y Choi, 2000; Varela, Rial y } \\
\text { García-Cueto, 2003; Wai-Ching y Gun-Fie, 2005; Aksu, 2006. }\end{array}$ \\
\hline $\begin{array}{l}\text { Instalaciones } \\
\text { adicionales }\end{array}$ & $\begin{array}{l}\text { Kandampully y Suhartanto, 2000; Chu y Choi, 2000; Wai-Ching y } \\
\text { Gun-Fie, 2005; Aksu, 2006; Ryan y Huimin, } 2007 .\end{array}$ \\
\hline $\begin{array}{l}\text { Ubicación } \\
\text { del hotel y } \\
\text { accesibilidad }\end{array}$ & $\begin{array}{l}\text { LeBlanc y Nguyen, 1996; Kandampully y Suhartanto, 2000; Varela, } \\
\text { Rial y García-Cueto, 2003; Wai-Ching y Gun-Fie, 2005; Ryan y } \\
\text { Huimin, 2007; Jonasson y Mård, } 2007 .\end{array}$ \\
\hline $\begin{array}{l}\text { Condiciones de } \\
\text { la habitación }\end{array}$ & $\begin{array}{l}\text { Peiró, Martínez-Tur y Ramos López, 1999; Chu y Choi, 2000; } \\
\text { Wai-Ching y Gun-Fie, 2005; Ryan y Huimin, } 2007 .\end{array}$ \\
\hline $\begin{array}{l}\text { Nivel de } \\
\text { servicio }\end{array}$ & $\begin{array}{l}\text { Kandampully y Suhartanto, } 2000 \text {; Chu y Choi, 2000; Varela, Rial y } \\
\text { García-Cueto, 2003; Wai-Ching y Gun-Fie, 2005; Ryan y Huimin, } \\
\text { 2007; Jonasson y Mård, } 2007 .\end{array}$ \\
\hline $\begin{array}{l}\text { Identidad } \\
\text { corporativa }\end{array}$ & Sanz de la Tajada, I994; LeBlanc y Nguyen, 1996. \\
\hline Reputación & $\begin{array}{l}\text { Chu y Choi, 2000; Wai-Ching y Gun-Fie, 2005; Ryan y Huimin, } \\
2007 .\end{array}$ \\
\hline $\begin{array}{l}\text { Opciones } \\
\text { de comida y } \\
\text { bebida }\end{array}$ & $\begin{array}{l}\text { Peiró, Martínez-Tur y Ramos López, I999; Kandampully y } \\
\text { Suhartanto, 2000; Chu y Choi, 2000; Wai-Ching y Gun-Fie, 2005; } \\
\text { Aksu, 2006; Ryan y Huimin, 2007; Jonasson y Mård, } 2007 .\end{array}$ \\
\hline
\end{tabular}

Fuente: Elaboración propia. 
En estudios dirigidos a la medición del componente cognitivo de la imagen turística de hoteles se plantea que los atributos del hotel guían la elección de los consumidores de un producto en oposición a otro (Chu y Choi, 2000; Díaz Armas, 2003: 30; Beerli, 2007: 6, 2008: I5). En el cuadro I mostramos los atributos utilizados con más frecuencia por los investigadores para medir la imagen de hoteles, los cuales son reflejados en la literatura turística especializada.

Muchos estudios de imagen utilizan un conjunto de atributos sin tomar en cuenta ningún criterio de agrupación. En este caso se encuentran los realizados por LeBlanc y Nguyen (1996: 32), Aksu (2006: I87), Ryan y Huimin (2007). Por otra parte, autores como Peiró, Martínez-Tur y Ramos López (1999) emplean criterios de agrupación difusos. En otras investigaciones, se han obtenido diferentes atributos constitutivos de la imagen de los hoteles, $y$ han sido agrupados con criterios particulares (Chu y Choi, 2000;Wai-Ching y Gun-Fie, 2005).

Al proceder a la revisión documental en la empresa, se puede considerar en lo fundamental estudios previos relacionados con la imagen del hotel y el manual de identidad, si existe, mientras que, por otra parte, tenemos el registro de quejas y sugerencias, documentación referida a la matriz DAFO (debilidades, amenazas, fortalezas y oportunidades) y áreas de resultados claves. Esto brinda información relativa a atributos propios de la realidad de la empresa, donde el manual de identidad ofrece la posibilidad de identificar la forma en que piensa su público interno, lo que constituye una base para la imagen a proyectar (Costa, 2003: 10). A su vez, la matriz DAFO establece debilidades y fortalezas a tener en cuenta en los atributos identificables de cada área, que se consideren clave para el éxito de la empresa. Desafortunadamente, para generar atributos, en muchas ocasiones sólo se cuenta con el registro de quejas y sugerencias; no obstante, de éste podemos extraer los atributos que los clientes consideran relevantes para su satisfacción.

Como parte de la fase cualitativa propuesta por Sanz de la Tajada (I994: 60 ), se lleva a cabo la entrevista a profundidad a una muestra preestablecida de empleados, quienes, desde la perspectiva de la oferta, pueden aportar importantes elementos que considerar dentro de la imagen del hotel. En este caso es necesario dividir las entrevistas entre los directivos e incluir empleados con disímiles ocupaciones para poder obtener la visión más amplia posible 
del problema a tratar. También se utiliza la observación directa dentro de la concepción cuantitativa de investigación, ésta se lleva de forma rigurosa por el investigador, prestando atención de manera premeditada a situaciones que puedan aportarle criterios sobre los atributos formativos de la imagen. Todo esto hace posible definir escalas de medida generando atributos y considerando toda la información manejada con anterioridad.

En este paso, el sistema empírico formado por elementos de las ciencias físicas y sociales es representado por un sistema abstracto en el cual se emplean números para interpretar esos elementos.Al determinar la imagen según los componentes, criterios y atributos que la integran, es posible asociar cada característica a una escala numérica. En este caso proponemos, como una escala principal, una escala ordinal tipo Likert de 5 , que permite discernir entre diferentes actitudes hacia un mismo objeto; estos números se utilizan como símbolos que modelan las características de interés en el sistema empírico.

Para dar continuidad a la elaboración del instrumento de medida, con base en la escala de medida generada, se lleva a cabo el paso 5, es decir, se diseña el instrumento de investigación: el cuestionario a partir del modelo conceptual de la imagen. El cuestionario incluye preguntas estructuradas para medir el componente cognitivo, holístico, la imagen en general, la satisfacción e intención de repetir la visita; además de preguntas no estructuradas para identificar el componente único y distintivo de la imagen del hotel. Esta fase culmina con el paso 6: pretest del cuestionario propuesto, donde se usan técnicas cualitativas, como la opinión de expertos y la dinámica de grupos; con la primera se busca valorar la adecuación del contenido de las preguntas a lo que se pretende medir, la segunda se emplea para determinar si es comprensible la redacción en general, entre otros aspectos esenciales para el éxito del siguiente paso; este paso termina con la aplicación de la encuesta a una muestra piloto de turistas.

En esta fase combinamos herramientas cuantitativas y cualitativas; utilizamos las técnicas cualitativas para estudiar posibles motivaciones como elemento para el descubrimiento de la imagen.Aunque la investigación cualitativa no muestra el valor relativo de cada elemento ni su participación en el proceso de decisión del cliente, sí permite realizar análisis de gran profundidad, donde se puede apreciar la dinámica de la realidad (explicativa del comportamiento y de las actitudes de los públicos objetivo). Una de las desventajas del empleo de este tipo de técnicas es que se usan en grupos que estadísticamente no son representativos de la población objeto de estudio. Debido a lo anterior, tales técnicas no han resuelto 
por completo el problema del conocimiento de actitudes y percepciones de los clientes de las empresas interesadas en estudios de imagen.

Sin embargo, el investigador cuenta con la posibilidad de emplear técnicas cuantitativas para desarrollar procedimientos que midan la imagen de las empresas. Éstas se aplican a grupos muestrales estadísticamente representativos de la población a estudiar. Aunque a este análisis se le cuestiona su relativa falta de profundidad y su visión estática de la realidad, posee un enfoque estructurado, que permite generalizar de forma objetiva las conclusiones extraídas de la investigación cuantitativa. Debido a las limitaciones metodológicas de las técnicas cualitativas y cuantitativas aplicadas por separado, Sanz de la Tajada ( 1994: 30 ) -entre otros, como Beerli, Martín y Moreno (2007: 6 y 2008: I 5)- avala el enfoque cualitativo-cuantitativo utilizado en este procedimiento, puesto que ofrece una respuesta más completa al problema.

Fase III.Trabajo de campo y presentación de los resultados de la investigación

Esta fase consta de tres pasos; comienza con el paso 7: recolección de la información, donde se emplea el cuestionario diseñado en el paso 5 y corregido en el paso 6; se aplica la encuesta personal definiéndose la unidad de muestreo, el tamaño de la muestra y el procedimiento de muestreo. Este paso es definitivo en la medición de la imagen percibida por el público objetivo, así como para conocer y prever la conducta o comportamiento de compra del cliente (Villafañe, 1993: I0; Trelles, 200 I:22). El paso 8: procesamiento e interpretación de la información, es el momento del estudio en que se usan técnicas estadísticas adecuadas a los objetivos de la investigación, las cuales permitirán corroborar las hipótesis planteadas. En consecuencia, se debe considerar un enfoque funcional teniendo en cuenta la medición de las percepciones-actitudes. Al referirnos al modelo explicativo del comportamiento de compra del turista, se precisó la importancia de las percepciones de cada cliente, que, combinadas con estímulos externos e internos, dan lugar a una actitud determinada que se concreta en la decisión de compra. Dentro del modelo de comportamiento del turista, propusimos que los criterios para incorporar y evaluar los hoteles del conjunto preliminar se centran esencialmente en los conceptos de utilidad/ valor relativo de los atributos (Hartline, Ross Wooldridge y Jones, 2003).

Como resultado de este análisis se dará prioridad a las percepciones, preferencias y actitudes, ya que hoteles con atributos percibidos adecuadamente son empresas con atributos preferidos. Estudios realizados sustentan que las 
expectativas y percepciones de los clientes durante el servicio recibido son muy importantes en la evaluación final de la experiencia de servicio (Wai-Ching y Gun-Fie, 2005). Los turistas pondrán a competir a cada hotel teniendo como base los beneficios particulares asociados a cada uno de ellos, a fin de hacerlos competitivos a la luz de ciertos atributos semejantes al valor percibido, y que establecen una regla de decisión en el momento de elegir (Carter y Fabricius, 2006: 27).

Teniendo en cuenta lo anterior surge la necesidad de referirse a la posibilidad de medición de la imagen de hoteles mediante una teoría multiatributo. Muchos autores consideran que la imagen puede investigarse y medirse con técnicas de medición de actitudes. Esta afirmación se fundamenta en que la imagen deviene una actitud (Costa, 2008: 5), concepto que incorporamos a esta investigación como línea de pensamiento a seguir. Por tanto, en la evaluación de las percepciones y actitudes, es imprescindible determinar los atributos que los individuos valoran para la formación de las imágenes respectivas sobre los hoteles considerados. A la hora de elegir el lugar de hospedaje, éstos forman parte de diferentes conjuntos de elección de cada turista (los que satisfacen necesidades psicológicas, más que fisiológicas); el papel determinante de atributos disímiles parte de su mapa de preferencias. Si el objetivo es medir imagen, lo es también medir actitud, de allí la posibilidad de aplicar la teoría multiatributos. Para ello, Fishbein (1967: 477) argumenta que existen dos componentes principales de las actitudes: el evaluativo (atributo del hotel) y la importancia dwe la creencia, es decir, lo importante que tiene para el cliente tal atributo para la satisfacción de sus necesidades y preferencias. Siguiendo a Chu y Choi (2000: 363), Pons (2000: 23), Wai-Ching y Gun-Fie (2005), se puede determinar la actitud hacia un destino u hotel, llevándolo a fórmula de la siguiente manera:

$$
A_{j}=\sum_{i=1}^{n} B_{i j} * a_{i}
$$

Donde:

$A j=$ Actitud hacia el hotel $\mathrm{j}$

$B i j=$ Intensidad de la creencia (atributo) $i$ en su oferta por parte del hotel $j$

$a i=$ Evaluación (importancia) de la creencia (atributo) $\mathrm{i}$

$n=$ Número de creencias (atributos) 
La teoría multiatributo posibilita medir y analizar la percepción de la imagen considerando la relevancia conferida a los atributos turísticos, así como la presencia relativa de éstos en un hotel específico, según el cliente (Chu y Choi, 2000:363;Wai-Ching y Gun-Fie, 2005). De tal suerte, una de las técnicas que ha alcanzado mayor auge debido a su gran poder explicativo y claridad expositiva es el análisis de importancia-valoración (Importance-Performance Analysis, IPA) también conocido como matriz mantilla (Martilla y James, 1977).

De acuerdo con esta matriz, es posible identificar estrategias en total consonancia con los preceptos básicos de la teoría multiatributo. En esta línea, varios estudios han puesto de manifiesto que no todos los atributos explican por igual la satisfacción global con un determinado servicio. Así, los consumidores suelen juzgar su rendimiento basándose únicamente en un número limitado de atributos (Ábalo Piñeiro, Varela Mallou y Rial Boubeta, 2006: 10), por lo que los atributos más importantes afectarán en gran medida la evaluación del usuario, mientras que los de menor relevancia apenas influirán en su valoración global (Ábalo Piñeiro,Varela Mallou y Rial Boubeta, 2006: 10). En resumen, la técnica estadística de tratamiento y análisis de la información debe ser multiatributo (Wai-Ching y Gun-Fie, 2005), y desembocar en una diversidad de componentes subjetivos y objetivos que obligue a medir atributos físicos y funcionales, por un lado, y psicológicos, por otro; además, debe considerar elementos holísticos, únicos y distintivos (Baloglu y McCleary, 1999; Pons, 2000: 23; García de los Salmones Sánchez, 2001:31).

Por último,y como colofón del procedimiento, está el paso 9: informe final del estudio de imagen en el hotel. Éste incluirá un resumen de las principales conclusiones derivadas de la medición de la imagen, así como la recomendación de líneas estratégicas generales a seguir.

\section{Discusión de los principales resultados}

Para comprobar las hipótesis propuestas, en la generación de los atributos que estructuran y determinan los componentes de la imagen global se empleó la revisión de bibliografía especializada, la observación directa, el libro de quejas y sugerencias, la matriz DAFO del hotel objeto de estudio, y se realizaron I 5 entrevistas en profundidad a trabajadores del hotel (como estudio cualitativo previo). Utilizamos el criterio de tres expertos, tres dinámicas de grupo, para pretestar el cuestionario inicialmente confeccionado. El uso apropiado de cada 
una de estas técnicas posibilitó definir el cuestionario adecuado para el logro de los objetivos de la investigación.

En el estudio se realizaron 120 encuestas personales en el Hotel Kohly, del 15 de abril al 18 de mayo de 2010 , con una confiabilidad de $95 \%$ utilizando el muestreo aleatorio simple.

El Hotel Kohly se encuentra ubicado en Av. 49 esquina 36A Reparto Kohly, Ciudad de La Habana. Es administrado por el Grupo de Turismo GAVIOTA S.A. y está dedicado al turismo internacional; es un hotel de ciudad, categoría tres estrellas, con I 36 habitaciones doble estándar, y vista al bosque, el mar o la ciudad.

\section{Componente único distintivo}

Como se observa en el cuadro 2 , de los turistas encuestados, $15.8 \%$ señala como característica distintiva del hotel ser ecológico; I $4.2 \%$, ser apartado, y $10.8 \%$, su diseño arquitectónico. El resto mencionó el ambiente familiar, la tranquilidad, el ambiente agradable y lo confortable, que demuestra lo difuso de este componente. En general podemos afirmar que no existe consenso en cuanto al atributo diferenciador del hotel; no obstante, en conjunto, estos atributos permiten diferenciar el Kohly del resto de los hoteles de tres estrellas de ciudad.

Cuadro 2. FreCuencia del COMPONENTE ÚNICO O DISTINTIVO

\begin{tabular}{lcc}
\hline & Frecuencia & Porcentaje \\
\hline Diseño arquitectónico & 13 & 10.8 \\
Ecológico & 19 & 15.8 \\
Apartado & 17 & 14.2 \\
Otros componentes & 71 & 59.2 \\
\hline Total & 120 & 100.0 \\
\hline
\end{tabular}

Fuente: Elaboración propia. 


\section{Componente afectivo}

En términos generales, para el componente holístico experimentado al visitar un hotel, la imagen del Hotel Kohly es favorable, como lo demuestra el cálculo de la moda (cuadro 3). Al analizar cada característica que describe el ambiente del hotel se observa que es calificado como agradable, excitante, novedoso y relajado.

CuAdro 3. Moda y Media del COMPONENTE holístico: ATMÓsFera o SENTIMIENTO EXPERIMENTADO AL VISITAR EL HOTEL

\begin{tabular}{lll}
\hline Ambiente & Media & Moda \\
\hline Desagradable-agradable & 4.4167 & 4.0000 \\
Inseguro-seguro & 4.5000 & 4.0000 \\
Tradicional-novedoso & 3.9417 & 4.0000 \\
Ruidoso-relajado & 4.4333 & 4.0000 \\
Componente holístico & 4.3229 & 4.0000 \\
\hline
\end{tabular}

Fuente: Elaboración propia.

\section{Componente perceptual-cognitivo}

Se realiza un análisis del componente perceptual-cognitivo a través de los criterios y atributos que lo integran. En el cuadro 4 se indica el grado de acuerdo o desacuerdo planteado por los encuestados respecto a éstos, así como la importancia que el cliente concede a cada uno de ellos.

Todos los atributos que se consideraron para medir el componente cognitivo de la imagen del Hotel Kohly fueron valorados como muy importantes por los turistas encuestados.

Los clientes dicen estar de acuerdo con el criterio de servicios generales, lo que se refleja claramente en el resultado de la moda; encontrando entre los atributos mejor valorados para el hotel, la rapidez del servicio, la buena apariencia de los empleados, la seguridad y los precios atractivos. Al utilizar la moda como estadígrafo decisivo principal, es evidente que la mayor cantidad de 
La imagen como recurso estratégico en la gestión de instituciones hoteleras: el caso del Hotel Kohly de la Ciudad de La Habana

CuAdRo 4. Modas y medias PARA CADA ATRIBUto DEL COMPONENTE PERCEPTUAL-COGNITIVO E IMPORTANCIA CONCEDIDA A CADA ATRIBUTO

\begin{tabular}{|c|c|c|c|c|}
\hline \multirow{2}{*}{ Criterios / atributos } & \multicolumn{2}{|c|}{ Acuerdo } & \multicolumn{2}{|c|}{ Importancia } \\
\hline & Media & Moda & Media & Moda \\
\hline Servicios generales & $4.47 \mid 2$ & 5 & 4.9363 & 5 \\
\hline $\begin{array}{l}\text { Empleados amables y de buena } \\
\text { apariencia }\end{array}$ & 4. 7500 & 5 & 5.0000 & 5 \\
\hline Servicio rápido & 4.3250 & 5 & 5.0000 & 5 \\
\hline Atención personalizada & 4.0750 & 4 & 4.8750 & 5 \\
\hline Eficiente servicio de registro y salida & 4.5417 & 5 & 4.9500 & 5 \\
\hline $\begin{array}{l}\text { Empleados con conocimientos } \\
\text { suficientes }\end{array}$ & 4.3417 & 4 & 4.8667 & 5 \\
\hline $\begin{array}{l}\text { Estructurado para atender } \\
\text { discapacitados }\end{array}$ & 4.1250 & 4 & 4.8833 & 5 \\
\hline Servicio corresponde con las estrellas & 4.8750 & 5 & 4.8833 & 5 \\
\hline Servicio adecuado a toda la familia & 4.3583 & 4 & 4.8583 & 5 \\
\hline Correspondencia calidad/precio & 4.3833 & 4 & 4.9917 & 5 \\
\hline Seguridad en todas las áreas & 4.7000 & 5 & 4.9917 & 5 \\
\hline Precios atractivos & 4.7083 & 5 & 5.0000 & 5 \\
\hline Habitaciones & 4.5736 & 5 & 4.9694 & 5 \\
\hline Cama, colchón y almohada cómodos & 4.5750 & 5 & 5.0000 & 5 \\
\hline Buena iluminación & 4.7750 & 5 & 4.9833 & 5 \\
\hline Habitaciones climatizadas & 4.8167 & 5 & 5.0000 & 5 \\
\hline $\begin{array}{l}\text { Habitaciones con televisión en línea } \\
\text { con el mundo }\end{array}$ & 4.1667 & 4 & 4.8417 & 5 \\
\hline Servicio de limpieza eficiente & 4.5167 & 5 & 5.0000 & 5 \\
\hline Habitaciones acogedoras & 4.5917 & 5 & 4.9917 & 5 \\
\hline Gastronomía, bebidas y recreación & 3.4095 & 5 & 4.9464 & 5 \\
\hline $\begin{array}{l}\text { Variedad y cantidad de la oferta } \\
\text { gastronómica y de bebidas }\end{array}$ & 4.1750 & 4 & 5.0000 & 5 \\
\hline Calidad de comidas y bebidas & 4.3333 & 5 & 5.0000 & 5 \\
\hline $\begin{array}{l}\text { El servicio de cafetería, pizzería y } \\
\text { restaurante se corresponde con sus } \\
\text { expectativas }\end{array}$ & 4.2583 & 5 & 4.9917 & 5 \\
\hline
\end{tabular}


CuAdro 4. Modas y Medias PARA CADA ATRIBUto del COMPONENTE PERCEPTUAL-COGNITIVO E IMPORTANCIA CONCEDIDA A CADA ATRIBUTO

(FINALIZA)

\begin{tabular}{|c|c|c|c|c|}
\hline \multirow{2}{*}{ Criterios / atributos } & \multicolumn{2}{|c|}{ Acuerdo } & \multicolumn{2}{|c|}{ Importancia } \\
\hline & Media & Moda & Media & Moda \\
\hline $\begin{array}{l}\text { Decoración y ambiente de bares } \\
\text { y restaurante }\end{array}$ & 4.5083 & 5 & 4.9833 & 5 \\
\hline Servicio de discoteca & 1.6250 & 1 & 4.8333 & 5 \\
\hline Actividades culturales & 2.6917 & 4 & 4.8750 & 5 \\
\hline Variedad de recreación & 2.2750 & I & 4.9417 & 5 \\
\hline Servicios e instalaciones adicionales & 4.5437 & 5 & 4.8906 & 5 \\
\hline $\begin{array}{l}\text { Salón de cibercafé para negocios, } \\
\text { telefonía y mensajería }\end{array}$ & 4.3667 & 4 & 4.8833 & 5 \\
\hline Servicios de gimnasio, sauna y masaje & 4.6167 & 5 & 4.8917 & 5 \\
\hline Surtido de las tiendas & 4.1917 & 4 & 4.8833 & 5 \\
\hline Ofertas de piscina & 4.6583 & 5 & 4.9083 & 5 \\
\hline Cancha de tenis, bolera y billar & 4.5583 & 5 & 4.9083 & 5 \\
\hline Buen servicio del consultorio médico & 4.6333 & 5 & 4.8833 & 5 \\
\hline Buró de turismo & 4.6583 & 5 & 4.9167 & 5 \\
\hline Punto de renta de autos, taxi y parqueo & 4.6667 & 5 & 4.8500 & 5 \\
\hline Condiciones del destino turístico & 4.4458 & 5 & 4.9444 & 5 \\
\hline Imagen y reputación del destino & 4.7417 & 5 & 5.0000 & 5 \\
\hline Destino seguro e iluminado & 4.3750 & 4 & 4.9833 & 5 \\
\hline $\begin{array}{l}\text { Carreteras en buen estado y } \\
\text { señalizadas }\end{array}$ & 4.1000 & 5 & 4.8583 & 5 \\
\hline Ofertas extrahoteleras & 4.3250 & 5 & 4.8417 & 5 \\
\hline Ubicación de la instalación & 4.5500 & 5 & 4.9917 & 5 \\
\hline Cuidado del medio ambiente & 4.5833 & 5 & 4.9917 & 5 \\
\hline Componente cognitivo & 4.2887 & 5 & 4.9374 & 5 \\
\hline
\end{tabular}

Fuente: Elaboración propia. 
los encuestados está de acuerdo con cada atributo de este criterio, otorgándole un valor de 4 o 5 .

En cuanto al criterio habitaciones, el común de los clientes está muy de acuerdo con cada atributo que lo integra, como demuestra el valor ( $\operatorname{moda}=5$ ). Aunque es necesario señalar que en el caso de habitaciones con televisión en línea con el mundo sólo se muestra de acuerdo con tal atributo (moda $=4$ ).

Los encuestados exponen su estado de muy de acuerdo respecto al criterio de gastronomía, bebidas y recreación, pero debemos anotar que en este caso es donde mayor diversidad de opiniones existen, puesto que el valor de la media es de 3.4095 , lo cual se debe fundamentalmente a la mala opinión sobre los servicios de discoteca y a la variedad en la recreación del hotel. No obstante las actividades culturales son valoradas con 4 en la moda, existe gran tendencia a valores negativos al respecto, como se ve en el resultado de la media.

Al analizar el criterio servicios e instalaciones adicionales se puede observar que la mayoría de los encuestados está muy de acuerdo con los atributos relacionados. Las condiciones del destino turístico mantienen la moda de 5, evidenciando que los turistas tienen un máximo grado de acuerdo con estos atributos, salvo la iluminación y la seguridad del destino con una moda de 4.

De manera general, el componente perceptual-cognitivo presenta una moda de 5 , lo que significa que es valorado muy positivamente.

Después de haber realizado el anterior análisis del componente perceptualcognitivo, estamos en condiciones de determinar los puntos fuertes y débiles del hotel desde la percepción del cliente a través de la matriz de análisis de importancia-valoración o matriz mantilla, detectando como puntos débiles: servicios de discoteca, actividades culturales y variedad recreativa; los demás atributos son calificados de fuertes. Éstos son puntos que la gestión del hotel debe considerar para trazar sus estrategias. Se hace necesario no descuidar los puntos fuertes para potenciarlos como ventajas competitivas, pues los clientes perciben estos atributos altos en importancia y su presencia en el servicio es positiva; y trabajar para eliminar los puntos débiles que reflejan baja valoración y alta importancia para los turistas en su concepción de la imagen del hotel. En este caso no existen, según la valoración de los encuestados, atributos de baja prioridad ni atributos exagerados. 


\section{Imagen general, cumplimiento de las expectativas, intención de repetir la visita}

La imagen general de un hotel es decisiva, pues influye en las motivaciones, percepciones, preferencias y actitudes de los clientes hacia él, por lo que a continuación se analizará aquélla en el hotel objeto de estudio.

Los encuestados, atendiendo al valor de la moda, plantean que la imagen general del Hotel Kohly es muy positiva, como se observa en el cuadro 5.

Haciendo un análisis del cumplimiento de las expectativas de los clientes, la moda indica que el valor más frecuente fue 5 , por tanto la mayor parte de los encuestados cree que sus expectativas se cumplieron totalmente (cuadro 5).

Si pretendemos realizar un análisis de la intención de los encuestados de repetir su visita al hotel, es preciso tener en cuenta las percepciones, hechos o experiencias resultado de la influencia de la imagen primaria que obtuvieron durante la visita, lo que ejerce una opinión determinante sobre el cliente potencial en la decisión de compra en un establecimiento de este tipo. Los encuestados manifiestan, predominantemente, la intención de repetir la visita, ya que el valor más frecuente fue 5 , reflejando la intención de volver con toda seguridad a la instalación. Lo antes descrito puede ser corroborado en el cuadro 5.

Cuadro 5. IMAgen General DEL HOtel, CUMPLIMIENTO

DE LAS EXPECTATIVAS, REPETICIÓN DE LA VISITA

\begin{tabular}{lcc}
\hline Percepción & Media & Moda \\
\hline Imagen general & 4.5667 & 5 \\
Cumplimiento de las expectativas & 4.5167 & 5 \\
Repetición de la visita & 4.5167 & 5 \\
\hline
\end{tabular}

Fuente: Elaboración propia.

\section{Segmentos del mercado objeto de estudio}

Teniendo en cuenta la edad, observamos que las personas que más visitan el hotel están en el rango de edad entre 25 y 35 años (35.8\%) y los que menos lo hacen son los mayores de 60 años (5.8\% solamente). En cuanto a la nacionalidad, encontramos que de los 120 encuestados el mercado emisor de mayor fuerza 
es Venezuela, con $46.7 \%$, siguiendo en ese mismo orden Ecuador con I $2.5 \%$ y Canadá con 10.8 \%; los de menor prevalencia son Chile y Panamá.

Dentro de los visitantes extranjeros, los hombres representan $51.7 \%$ del total; el resto está compuesto por mujeres. Si nos referimos al motivo por el cual se realiza la visita se puede apreciar que el de mayor peso es el trabajo, con $36.7 \%$, seguido de descanso y recreación, con 20.8 y $20 \%$ respectivamente.

Según el criterio de frecuencia de la visita, podemos afirmar que la mayoría de los encuestados visita el hotel por primera vez, luego el mayor porcentaje corresponde a los que han visitado la instalación en dos o tres ocasiones, $y$, por último, en menor frecuencia, se encuentran los que lo han hecho en cuatro o más oportunidades.

\section{Relaciones entre imagen general, satisfacción e intención de repetir la visita}

Para establecer relaciones entre variables se recurrió a la correlación estadística, donde el coeficiente de correlación describe la relación lineal básica entre dos variables. En este caso usaremos el coeficiente de Spearman para datos de naturaleza cualitativa que responden a una escala de medida ordinal.

En el caso del cuadro 6 la correlación entre la imagen general y la satisfacción presenta una tendencia a la correlación media, aunque el nivel de confianza con que se muestra este resultado sólo alcanza $90 \%$. Por otra parte, la correlación entre la imagen general y la intención de repetir la visita es media, con un nivel de confiabilidad de $100 \%$.

Cuadro 6. Coeficiente de correlación de Spearman para la imagen general CON LA INTENCIÓN DE REPETIR LA VISITA Y LA SATISFACCIÓN

\begin{tabular}{lcc}
\hline Rho de Spearman & $\begin{array}{c}\text { Intención de } \\
\text { repetir la visita }\end{array}$ & $\begin{array}{c}\text { Cumplimiento } \\
\text { de las expectativas }\end{array}$ \\
\hline $\begin{array}{l}\text { Percepción de la imagen } \\
\text { general }\end{array}$ & 0.352 & 0.152 \\
Sig. (bilateral) & 0.000 & 0.097 \\
\hline
\end{tabular}

Fuente: Elaboración propia. 


\section{Lineamientos estratégicos para la imagen del Hotel Kohly}

Un análisis de los resultados basados en la matriz de análisis de importancia-

valoración o matriz mantilla nos dice que se debe trabajar manteniendo los puntos fuertes encontrados y concentrarse en la solución de los débiles. Como un resultado positivo podemos señalar que no se hallaron elementos posiblemente exagerados, lo que significa que no se están invirtiendo recursos en algo que no es valorado por los clientes.

Con el objetivo de lograr una imagen positiva del hotel, que se pueda sostener en el tiempo, lo que redundará en un alto nivel de satisfacción que garantice la repetición de la visita por parte de los huéspedes, se trazan las siguientes acciones:

I. Mantener el desempeño alcanzado en las dimensiones de servicios generales, habitaciones, servicios e instalaciones adicionales.

2. Abogar ante los responsables del mantenimiento de las condiciones del destino turístico para que se conserven y se perfeccionen.

3. Ampliar el número de ofertas culturales, brindando servicios de discoteca como medio para diversificar las actividades recreativas.

4. Mantener en las dimensiones de gastronomía, bebidas y recreación el buen desempeño alcanzado, identificadas por los siguientes atributos:

- Variedad y cantidad de la oferta gastronómica y de bebidas

- Calidad de comidas y bebidas

- El servicio de cafetería, pizzería y restaurante se corresponde con sus expectativas

- Decoración y ambiente de bares y restaurante.

5. Potenciar la distinción de un componente único que diferencie el hotel del resto de los semejantes; en este caso, ser ecológico podría ser esa característica, ya que la mayoría de los encuestados la destacan y se corresponde con las preferencias internacionales.

6. Implementar un diseño del servicio que sustente un ambiente agradable, relajado, novedoso y seguro.

7. Establecer un programa de promoción internacional basado en la descripción de los componentes de la imagen del Hotel Kohly. 
8. Adecuar la oferta al segmento de mercado de turistas que se encuentren en edades comprendidas entre 25 y 45 años, segmento latinoamericano, con motivaciones de compra relacionadas con trabajo, descanso y recreación, puesto que constituyen la mayor afluencia a la entidad.

\section{Conclusiones}

I. La imagen es un recurso intangible con que cuenta la empresa turística para desarrollar sus capacidades, la cual, al tratarse como una variable de la administración, puede convertirse en una ventaja sostenible capaz de reinventar el servicio ofertado, considerando las necesidades cambiantes de la demanda, lo que contribuye al empleo eficiente $y$ eficaz de los recursos.

2. Partiendo de investigaciones de carácter cualitativo y cuantitativo y del marco teórico-metodológico establecido, podemos concluir que el procedimiento a aplicar incluye de forma coherente y dinámica todos los componentes constitutivos de la imagen de hoteles, permitiendo medirla en el Hotel Kohly.

3. El componente afectivo del hotel objeto de estudio se caracteriza por un ambiente agradable, seguro, novedoso y relajado.

4. El área de la variedad recreativa no contribuye de manera positiva a la imagen general del hotel, puesto que los huéspedes le atribuyen a esta esfera un alto nivel de importancia, pero no perciben buenos resultados en este sentido.

5. En el caso del Hotel Kohly, mediante la aplicación del cuestionario a turistas, podemos definir su imagen general como muy positiva.

6. La mayoría de los encuestados opina que sus expectativas al visitar el hotel se cumplieron totalmente. Además, expresan que repetirín la visita con toda seguridad, como consecuencia de una correlación media con la percepción de la imagen general del Hotel Kohly, relación ésta de un alto grado de significación. 


\section{FUENTES CONSULTADAS}

Ábalo Piñeiro, J., J. Varela Mallou y A. Rial Boubeta (2006). "El análisis de importancia-valoración aplicado a la gestión de servicios".

Psicothema, 35 (4), 10.

Akin,A. (2006)."Gap Analysis in Customer Loyalty:A Research in 5-Star Hotels in the Antalya Region of Turkey”. Quality \& Quantity, 40, I87-205.

Baloglu, S. y D. Brinberg (1997). "Affective Images of Tourism Destination”. Journal of Travel Research, 35 (4), I I-I5.

Baloglu, S.y K.W. McCleary ( 1999). “A Model of Destination Image Formation”. Annals of Tourism Research, 26 (4), 868-897.

Barsky, J. y L. Nash (2003). "Customer Satisfaction: Applying Concepts to Industry-wide Measures”. Cornell Quarterly Articles, 35 (4).

Beerli,A., S. Martín y S. Moreno (2008). La concepción sistémica de la imagen del destino: una aproximación desde la imagen del alojamiento [en línea]. Disponible en: http://www.esade.es/cedit2004/pdfs/4I_Moreno.pdf [2008, 27 de mayo].

Carter, R. y M. Fabricius (2006). "Introduction to Destination Management”. World Tourism Organization Seminar,Addis Ababa, 27-29 de marzo.

Chen, N. y H. Pan (2006). Gothenburg's Image as a Tourism Destination among Typical Tourists and Sport Tourists. Tesis de maestría. Gotemburgo: Gothenburg University.

Chu, R. K. S. y T. Choi (2000). "An Importance-performance Analysis of Hotel Selection Factors in the Hong Kong Hotel Industry:A Comparison of Business and Leisure Travellers". Tourism Management, 2 I (4), 363-377. Comité Ejecutivo del Consejo de Ministros (2007). Decreto No. 28I. I6 de agosto de 2007.

Costa,J.(2003).“De la economía de producción a la economía de información”. Razón y Palabra, 34, 10.

(2008). El concepto del término imagen. Disponible en: www.rrppnet. com.ar/imagencorporativares.htm [2008, 27 de mayo].

Díaz Armas, R. (2003). Interrelación entre imagen y comunicación en destinos turísticos. Tesis doctoral. Tenerife: Universidad de La Laguna. 
Duffus Miranda, D. (2009). Procedimiento para medir la imagen de los hoteles de la Región Central: caso Hotel Los Caneyes. Tesis de maestría. Santa Clara, Cuba: Universidad Central “Marta Abreu” de Las Villas.

Echtner, C. M. y J. R. Ritchie (1993). “The Measurement of Destination Image: An Empirical Assessment”. Journal of Travel Research, 4, 3-I3.

Fishbein, M. (1967). "Attitude and the Prediction of Behavior", en M. Fishbein (ed.). Readings in Attitude Theory and Measurement. Nueva York:Wiley, 477-492.

Gándara, J. M., E. Torres Bernier y J. E. Costa Mielke (2007). La imagen de los destinos turísticos urbanos. Disponible en: www.gestionturistica.cl/ publicaciones/revistas [2007, 17 de diciembre].

García de los Salmones Sánchez, M. del M. (200l). La imagen de empresa como factor determinante en la elección de operador: identidad y posicionamiento de las empresas de comunicaciones móviles. Tesis doctoral. Santander: Universidad de Cantabria.

Hartline, M. D., B. Ross Wooldridge y K. C. Jones (2003)."Guest Perceptions of Hotel Quality: Determining Which Employee Groups Count Most”. Cornell Hotel and Restaurant Administration Quarterly, 5, 43-44.

Jonasson, P.y A. Mård (2007). Controlling Word-of-Mouth:A Qualitative Study of the Hotel Industry in the Region of Jönkoping. Tesis de maestría. Alemania. Kandampully, J. y D. Suhartanto (2000). "Customer Loyalty in the Hotel Industry:The Role of Customer Satisfaction and Image". International Journal of Contemporary Hospitality Management, I2 (6), 346-35 I.

LeBlanc, G. y N. Nguyen (1996). "An Examination of the Factors that Signal Hotel Image to Travellers". Journal of Vacation Marketing, 3 (I), 32-42.

Leech, N., K. Barret y G. Morgan (2005). SPSS for Intermedia Statistic. Aplication and Interpretation. Londres: LEA Publishers.

Martilla, J. A. y J. C. James (1977). "Importance-Performance Analysis. An Easily Applied Technique for Measuring Attribute Importance and Performance Can Further the Development of Effective Marketing Programs". Journal of Marketing, enero, 77-79.

Peiró,J. M.,V. Martínez-Tur y J. Ramos López (1999). “El triángulo de la calidad de servicio: una aproximación psicosocial”. Papeles del Psicólogo, 74, I8-24. 
Pérez Villanueva, O. (2004). Reflexiones sobre la economía cubana. La Habana: Ciencias Sociales.

Pons, R. (2000). Cuba como destino turístico de "Sol y playa": Imagen y posicionamiento.Tesis doctoral. Madrid: Universidad Complutense de Madrid.

Pons, R. y M. L. Morales (2008). "La elección de un destino por parte del potencial turista”. IV Conferencia de Ciencias Empresariales. Villa Clara, Cuba.

Ryan, C. y G. Huimin (2007). "Perceptions of Chinese Hotels (International Focus)". Cornell Hotel \& Restaurant Administration Quarterly, 48 (4), 380-391.

San Martín Gutiérrez, H. (2005). Estudio de la imagen de destino turístico y el proceso global de satisfacción: adopción de un enfoque integrador. Tesis doctoral. Santander: Universidad de Cantabria.

Sanz de la Tajada, L. (1994). Integración de la identidad y la imagen de la empresa: Desarrollo conceptual y aplicación práctica. Madrid: ESIC.

Varela, J., A. Rial y E. García-Cueto (2003). "Presentación de una escala de satisfacción con los servicios sanitarios de atención primaria”. Psicothema, I5 (3), 656-66I.

Wai-Ching, P. y D.Y. Gun-Fie (2005). “Comparing Satisfaction Levels of Asian and Western Travellers Using Malaysian Hotels". Journal of Hospitality and Tourism Management, 12 (I), 64-79. 
